\title{
Polnische Richter des alten österreichischen Verwaltungsgerichtshofes in der Gerichtsbarkeit der Zweiten Polnischen Republik (1918-1939)
}

\begin{abstract}
The emergence of independent Poland in November 1918 gave rise to an urgent need to rapidly construe Poland's own judiciary, including that of administrative type. In February 1919 the Supreme Court in Warsaw took over the competence of the Vienna Administrative Tribunal. All former judges of the latter: Wilhelm Binder, Roman Moraczewski, Włodzimierz Orski, Rudolf Różycki, Zbigniew Smolka became members of the Fourth Chamber of the Supreme Administrative Court. Jan Sawicki was appointed to the Chamber. The governmental Bill on the implementation of the Supreme Administrative Tribunal, prepared in 1922, was fully based on the Austrian law on Administrative Tribunal of 1875, Jan Sawicki being the author of the Bill. Thus the judges with the Austrian legal background occupied a prominent position in the newly formed Supreme Administrative Tribunal (NTA). Jan Sawicki became the first President of the NTA. For the twenty-two judges who made up the first team of the NTA over half derived from the former Austrian partition territory. Jan Sawicki performed the function of the NTA First President until 1928 when, for political reasons, he retired. Then - for over two years - it was R. Różycki who acted in the capacity of the one fulfilling the duties of the First President. He was eventually nominated to the position of the NTA First President in 1930. He performed this function until 1932 when he retired. It is worthwhile to note that from November 1926 R. Różycki was also the President of the Comeptence Tribunal. Also W. Orski was the first President of the Supreme Administrative Tribunal and he performed this function for less than one year (19331934). The dismissal of Jan Sawicki overnight (!) through applying urgent procedure to which the authoritarian regime referred to as "Sanacja" resorted, was reflective of the beginning of the tendency to limit the NTA independence. The decree of the President of 1932 on the NTA introduced new principles according to which the judges were expected to be appointed. It was Prime Minister who was vested in the exclusive right to indicate the candidates for the management posts in the Tribunal. The candidate for the President was not even required to be a NTA judge! The transitory provisions contained in the decree provided the government with the right to remove inconvenient judges and replace them with the individuals who proved to be friendly-disposed toward the "Sanacja" authorities. It is worthwhile to emphasize however that these provisions were exploited on a fairly limited scale and as result had no negative impact on the NTA judicial decisions.
\end{abstract}

\section{Galizien als juristische}

\section{Kaderschmiede des wiedergegründeten Polen}

Als im November 1918 Polen als unabhängiger Staat wieder erstand, erforderte dies den raschen Aufbau des Gerichtswesens mit eigenem juristischen Personal. Eine dezidiert polnische Rechtsprechung gab es nur in Galizien, wo seit 1869 das Polnische in der Verwaltung, dem Schulwesen und eben der Gerichtsbarkeit verwendet wurde. Im ehemals preußischen Teilungsgebiet dominierte das deutsche Element (Ende 1918 waren in diesem Bereich gerade 
einmal drei Polen angestellt). Daher gelangten nach Absprache zwischen dem Justizminister und den Gerichtspräsidenten der Berufungsgerichte von Krakau [Kraków] und Lemberg [Lwiw, Lwów] 1920 achtzig Richter aus dem vormaligen Galizien nach Großpolen. ${ }^{1}$ Schwierigkeiten ergaben sich auch bei der Besetzung des Warschauer Obersten Gerichtes, das ebenfalls konsequenterweise von Polen, die aus dem ehemals österreichischen Teil stammten, dominiert wurde.

Mit dem Dekret von Staatsoberhaupt Józef Piłsudski vom 8. Februar 1919 übernahm das Oberste Gericht in Warschau [Warszawa] die Kompetenzen des Wiener Verwaltungsgerichtshofes. ${ }^{2} \mathrm{Zu}$ diesem Zwecke wurde eine vierte Kammer des Obersten Gerichtes ins Leben gerufen, in der sich sämtliche Polen des österreichischen Verwaltungsgerichtshofes wiederfanden: Wilhelm Binder, Roman Moraczewski, Włodzimierz Orski, Rudolf Różycki und Zbigniew Smolka. Die Kammerpräsidentur hatte Jan Sa-

${ }^{1}$ GULCZYŃSKI, Mitglieder des Obersten Gerichtshofes 84. Über die personellen Schwierigkeiten im Gerichtswesen in den ersten Jahren der Unabhängigkeit schrieb 1929 S. Gołąb. Professor an der JagiellonenUniversität und Mitglied der Kodifikationskommission: „Denn wirklich , professionelle' Richter gab es nur im österreichischen Teil [...] Die Richter aus dem sogenannten Kleinpolen konnten allerdings - zahlenmäßig - nicht den diesbezüglichen Mangel in ganz Polen wettmachen" (GOŁĄB, in: Czasopismo Prawnicze i Ekonomiczne). Nichtsdestoweniger waren die Richter im ehemals russischen Teilungsgebiet meistens polnischer Herkunft.

${ }^{2}$ Siehe das Dekret zur Höchstgerichtsverfassung [ $w$ przedmiocie ustroju Sadu Najwyższego] vom 8. Februar 1919 (Dz.U. nr. 5, poz. 199) sowie das Dekret zu Änderungen in Rechtssprechungsämtern im ehemals österreichischen Teilungsgebiet $[w$ przedmiocie zmian $o$ urzadzeniach wymiaru sprawiedliwości $w b$. zaborze austriackim] vom 8.2.1919 (Dz.U. nr. 5, poz. 200). In Übereinstimmung mit dem Inhalt dieses letzten Erlasses übernahm das Oberste Gericht in Warschau neben den Aufgaben des Verwaltungsgerichtshofes auch die Kompetenzen des Obersten Gerichtshofes (für Zivil- und Strafsachen). wicki inne, der sich am 1. Dezember 1918 den polnischen Organen zur Verfügung gestellt hatte. Alle sechs befanden sich dann auch später in der ersten Zusammensetzung des Obersten Verwaltungsgerichtshofes [Najwyższy Trybunat Administracyjny]. Die Verwaltungskammer des Obersten Gerichtes befasste sich mit Beschwerden gegen Verwaltungsentscheidungen, allerdings ausschließlich für das Gebiet des ehemaligen Galizien. ${ }^{3}$

Für den vormals preußischen Teil wurde nämlich das (abgeänderte) Modell der Verwaltungsgerichtsbarkeit mit drei Instanzen beibehalten (das bis 1939 Bestand hatte), und die Kompetenzen des Preußischen Oberverwaltungsgericht in Berlin wurden bis zur Einrichtung des OVwGH 1922 vom Berufungsgericht in Posen übernommen. ${ }^{4}$ In den Anfangsjahren der wiedererstandenen Polnischen Republik wirkten die Verwaltungsgerichte also zweigleisig und vollkommen unabhängig voneinander. Darüber hinaus fußten Organisation und Kompetenzbereiche auf unterschiedlichen Rechtsgrundlagen. ${ }^{5}$ Der $\mathrm{Zu}$ -

\footnotetext{
${ }^{3}$ Interessanterweise überging das Dekret vom 8. 2. 1919 vollständig die Übernahme jener Kompetenzen, die zuvor das österreichische Reichsgericht inne gehabt hatte, somit jener Verwaltungsklagen, welche die verfassungsrechtlich geschützten bürgerlichen Grundrechte berührten. Inwieweit es sich hier um ein Versehen oder bewusstes Vorgehen handelt, lässt sich schwerlich feststellen. Man kann nur vermuten, dass ein derartiger Rechtsschutz außer Acht gelassen wurde, da der polnische Staat noch keine eigene Verfassung hatte. Als Folge wurde der Sachzuständigkeitsbereich der Kammer IV des Obersten Gerichtshofes beschränkt. Zum Thema der Rechtsprechung des k.k. Reichsgerichtes und Veraltungsgerichtshofes vgl.: DZIADZIO, Angelegenheiten aus Galizien $475 f$.

4 TARNOWSKA, Sądownictwo administracyjne 423; TARNOWSKA, Niedere Verwaltungsgerichtsbarkeit.

${ }^{5}$ Die ererbte preußische Gerichtswesen kannte drei Instanzen, wobei die Gerichte der ersten und zweiten Instanz - unter Teilhabe eines staatsbürgerlichen Elements - Revisionsurteile fällten, wogegen die österreichische Verwaltungsgerichtsbarkeit ausschließlich aus Berufsrichtern bestand, bloß eine In-
} 
ständigkeitsbereich der örtlichen vierten Kammer des OVwGH war noch in anderer Hinsicht beschränkt: Im ehemalig russischen Teil gab es überhaupt keinen verwaltungsgerichtlichen Rechtsschutz. Daher war es eine der dringendsten Aufgaben der politischen Führung, eine einheitliche landesweite Verwaltungsgerichtsbarkeit zu organisieren.

\section{Jan Sawicki,}

\section{Vater des Gesetzes zum Obersten Verwaltungsgerichtshof von 1922}

Es verwundert daher nicht, dass die Bildung eines einheitlichen Ressorts für Verwaltungsgerichte im Vorfeld der Beschlussfassung für die Märzverfassung von 1921 durch den Sejm hitzig diskutiert wurde. Es gab jedoch wenig Befürworter des österreichischen Modells. Interessanterweise rekrutierten sich die Gegenstimmen vorrangig aus der galizischen Wissenschaftsund Juristenszene, welcher die Nachteile am besten bekannt waren, als da waren Säumigkeit und Langwierigkeit der Verfahren. Galizien war es auch, wo die ersten Entwürfe für die Organisation einer Verwaltungsgerichtsbarkeit entstanden, die eine doppelte Instanz im Verwaltungsverfahren veranschlagte und die Urteilsmöglichkeiten zu reformieren gedachte. ${ }^{6}$

Der einzige eigentliche Verteidiger des österreichischen Verwaltungsgerichtshofes war seinerzeit Jan Sawicki, der 1920 sein eigenes Modell

stanz hatte und Urteile nur aufheben konnte. Der Zuständigkeitsbereich von preußischen Verwaltungsgerichten fußte auf dem Enumerationsprinzip, der von österreichischen auf einer Generalklausel. Vgl.: OLECHOWSKI, Europäische Modelle 147-149.

${ }^{6}$ Z.B. der Entwurf zur Organisation der Verwaltungsgewalt der Juristischen Gesellschaft Lemberg von 1919 bzw. der Vorschlag von Kazimierz W. Kumaniecki, Verwaltungsrechtsexperte und Prof. der Jagiellonen-Universität. Vgl.: MALEC, Najwyższy Trybunał Administracyjny 23.; MALEC, Oberster Verwaltungsgerichtshof. präsentierte. Die Umstände der Publikation im Amtsblatt des Justizministeriums sind in diesem Zusammenhang nicht uninteressant. Der direkte Anlass für J. Sawicki, mit einem eigenen Entwurf zur Organisation von Verwaltungsgerichten aufzutreten, war ein zuvor veröffentlichter Artikel, dessen Autor für eine Abschaffung selbiger plädierte und deren Fälle durch allgemeine Gerichte behandelt wissen wollte. Für eine derartige Vorgehensweise bei strittigen Verwaltungssachen sprach auch, dass der junge Staat weder über eine probate Richterschaft noch über die finanziellen Mittel verfügte, um eine tiefgreifende Reform in der Verwaltungsgerichtsbarkeit in Angriff nehmen zu können. J. Sawicki griff in seiner Verteidigung für die Beibehaltung selbiger das Spezielle von Verwaltungsstreitsachen vor dem Hintergrund der unterschiedlichen Funktion des Zivilrechts auf. Er teilte auch nicht die Ansicht, die Polnische Republik könne sich die Einrichtung einer gesonderten Verwaltungsgerichtsbarkeit nicht leisten, indem er detailliert die Unterhaltskosten der Strukturen des österreichischen Verwaltungsgerichtshofes darlegte und nachweisen konnte, dass eine adäquate Einrichtung mit entsprechend weniger Personal nicht nur nicht die Staatsfinanzen belasten würde, sondern - im Gegenteil - dem Staatswohl und dem gedeihlichen Fortkommen der Republik dienlich sei. ${ }^{7}$

Daher schlug Sawicki unter Berücksichtigung der schwierigen Lage die Einrichtung eines Gerichtes für Streitfragen des Öffentlichen Rechts vor, das als eigenständige Institution vom Obersten Gericht getrennt wäre und dessen Richter sich je zur Hälfte aus Beamten sowie aus Richtern und Anwälten zusammensetzen würden. Diese Überlegungen fasste er in einem Vorschlag zusammen: „Gesetzesentwurf über die Einsetzung eines Reichsgerichts als Verwal-

\footnotetext{
${ }^{7}$ SAWICKI, Sądy administracyjne.
} 
tungs-, Wahl- und Kompetenzgericht(shof)“ .8 Als Grundlage seines Entwurfes übernahm er die gesetzlichen Vorschriften über die Einsetzung des Verwaltungsgerichtshofes von 1875 und ergänzte diese um jene über die Einsetzung des Reichsgerichts von 1867 hinsichtlich der Funktion als Kompetenzgericht. Sawickis Vorschlag war also nichts anderes, als die Anpassung des österreichischen Verwaltungsgerichtshofgesetzes an die polnischen Realien (Art. 4 der Vorlage entsprach $\S 3$ des Gesetzes; Art. 5 war gleich $\S 2$, Art. 17 entsprach $\S 7$ und Art. 11 war fast identisch mit $\S 6) .{ }^{9}$

Da die Stimmen der Praktiker wie auch jene von Seiten der Wissenschaft der österreichischen Verwaltungsgerichtsbarkeit mit nur einer Instanz skeptisch gegenüberstanden, sprach sich die erste polnische Verfassung vom 17. März 1921 für eine komplexere Struktur der Verwaltungsgerichte aus. In Art. 73 sah sie die Einrichtung einer Verwaltungsgerichtsbarkeit mit mehreren Instanzen vor, die unter einem Obersten Verwaltungsgerichtshof auf einem Zusammenwirken des bürgerlichen und richterlichen Ele-

\footnotetext{
8 „Projekt ustawy o ustanowieniu Trybunału Państwa jako sadu (trybunatu) administracyjnego, wyborczego i kompetencyjnego"

${ }^{9}$ Art. 11 der Vorlage entsprach vollkommen $\S 6$ des österreichischen Gesetzes von 1875. Es legte nämlich fest: „Der Verwaltungsgerichtshof hat in der Regel auf Grund des in der letzten administrativen Instanz angenommenen Thatbestandes $\mathrm{zu}$ erkennen. [Sachverhalte und Beweismittel, die erst nach Ergehung einer angefochtene Entscheidung oder Verfügung eingebracht werden, werden vom Gerichtshof nicht berücksichtigt]. Findet jedoch der Verwaltungsgerichtshof, daß der Thatbestand actenwidrig angenommen wurde, oder daß derselbe in wesentlichen Punkten einer Ergänzung bedarf, oder daß wesentliche Formen des Administrativverfahrens außer Acht gelassen worden sind, so hat er die angefochtene Entscheidung oder Verfügung wegen mangelhaften Verfahrens aufzuheben und die Sache an die Verwaltungsbehörde zurückzuleiten, welche die Mängel zu beheben und hierauf eine neue Entscheidung oder Verfügung zu treffen hat."
}

ments basieren sollte. Die Verfassungsbestimmung berücksichtige eher das preußische als das österreichische Modell. Allerdings war die Verfassung inhaltlich eher ein Spiegelbild idealistischer Staatsvorstellungen als des status quo, der von einer politischen oder auch wirtschaftlichen Stabilität weit entfernt war. Daher musste die Regierung bei der Formulierung der Verordnungen zur Verfassung die Realien in einem größeren Maße berücksichtigen.

Sie entschied sich daher 1922 nicht zu einer vollständigen Umsetzung von Art. 73 der Verfassung und entschloss sich zu einem Kompromiss: Die Einrichtung niederer Verwaltungsgerichte wurde aufgeschoben, der Oberste Verwaltungsgerichtshof jedoch ins Leben gerufen. Aufgrund des fehlenden Archivmaterials sind die genauen Vorgänge in der Regierung nicht mehr zu eruieren; klar ist jedoch, dass J. Sawicki Mitglied jener Kommission war, die vom Justizminister eingesetzt wurde, um die Gesetzesvorlage zur Gründung des OVwGH vorzubereiten. ${ }^{10}$ Die Regierung wird wohl vor der Kommission nicht verborgen haben, dass mit dem Aufbau einer kohärenten öffentlichen Verwaltung (auf Regierungssowie gebietskörperschaftlicher Ebene) und der damit verbundenen Struktur von Verwaltungsgerichten bis zu einer wesentlichen Verbesserung der internen Situation im Staate zugewartet werden musste. Das ließ den Vorschlag Sawickis von vor zwei Jahren bezüglich eines Gerichtshofes in einer Instanz attraktiver erscheinen. Die Regierung stimmte seinem Gesetzesentwurf zu, die sodann als Regierungsvorlage im Februar 1922 in den Sejm gelangte. J. Sawicki hatte in diesem „neuen“ Entwurf bloß eine weitere Überarbeitung der Artikel vorgenommen bzw. Bestimmungen entfernt, die den Gerichtshof zu Entscheidungen in Kompetenzkonflikten zwischen Verwaltung und Gerichte

\footnotetext{
${ }^{10}$ Gazeta Sądowa Warszawska, Nr.43 vom 25. 10. 1937.
} 
berechtigt hätten. Die März-Verfassung sah nämlich hierfür die Einrichtung eines speziellen Kompetenzgerichtshofes [Trybunat Kompetencyjny] vor. Dieser nahm 1926 seine Tätigkeit auf. Die Regierung empfahl dem Sejm somit das österreichische VwGH-Modell als das vorteilhafteste hinsichtlich der Möglichkeiten des Staates und der Bürgeransprüche. ${ }^{11}$ Die SejmKommission bewertete die Argumentation als „höchstgradig gerechtfertigt”.

Die Vorlage zum Durchführungsgesetz über den Obersten Verwaltungsgerichtshof von 1922 basierte zur Gänze und fast spiegelbildlich auf dem österreichischen von 1875. Es wurde am 3. August 1922 vom Sejm beschlossen. Sawickis Vorlage erlangte vor allem deshalb Zustimmung, da wegen fehlender qualifizierter Richter die Umsetzung der Verfassungsbestimmungen nicht möglich gewesen wäre. Dieses wesentliche Hindernis bei der Schaffung neuer verwaltungsgerichtlicher Strukturen war Sawicki bereits 1920 klar gewesen. Überzeugend hatte er seinerzeit dargelegt, dass die Einsetzung des

\footnotetext{
${ }^{11}$ Außerdem wurden zur Begründung der polnischen Vorlage zum Verwaltungsgerichtgesetz zu einem Gutteil die Argumente der österreichischen Regierung von 1875 ausgegraben. Man muss sich hier in Erinnerung rufen, dass in Österreich der Beschlussfassung über die Einrichtung eines Verwaltungsgerichtshofes eine Debatte vorangegangen war, in der Stimmen nach einer Verwaltungsreform nach preußischem Vorbild laut geworden waren. Außerdem wurde das österreichische Gesetz teilweise als verfassungswidrig angesehen. Darüber hinaus ergab sich die mangelnde Übereinstimmung des polnischen Gesetzes von 1922 mit der Verfassung nicht bloß in der Akzeptanz fehlender niederer Instanzen, sondern auch dadurch, dass die März-Verfassung auch einen Schutz der Rechtsordnung durch die Verwaltungsgerichtsbarkeit vorsah, das Gesetz dagegen nur den Schutz öffentlicher subjektiver Bürgerrechte. Vgl. Motivenbericht zu dem Gesetzentwurfe, betreffend die Errichtung eines Verwaltungsgerichtshofes. in: KASERER, Gesetz; mit Begründung der polnischen Gesetzesvorlage: AAN, Protokoły posiedzeń Rady Ministrów [Ministerratsprotokolle], Bd. 17, Mikrofilm Nr. 20061, 311.
}

OVwGH nach österreichischem Vorbild nicht allzu teuer ausfallen würde, da sie bloß in der Neuorganisation bestehender verwaltungsgerichtlicher Strukturen bestünde. Darüber hinaus würden die Beamten des jungen Staates auf einem bedeutenden Teil des Staatsgebietes nur einer Instanz mit Kassationsrecht unterstehen, was unter den schwierigen Nachkriegsbedingungen eine gewisse Selbstständigkeit bei der Handhabung öffentlicher Aufgaben gewährleisten würde. Nicht ganz bedeutungslos war auch das Vertrauen der Regierung in die Erfahrung der vormaligen VwGH-Richter, die bereits seit 1919 erfolgreich die Bestimmungen des österreichischen Gesetzes umsetzten. Die Beibehaltung dieses gerichtlichen und verwaltungstechnischen Schutzmechanismus' sicherte den Richtern österreichischer Provenienz auch Schlüsselpositionen im neuen Obersten Verwaltungsgerichtshof.

\section{Jan Sawicki, Rudolf Różycki und Włodzimierz Orski an der Spitze des Obersten Verwaltungsgerichtshofes}

Erster Präsident des OVwGH wurde Jan Sawicki. ${ }^{12}$ Als Präsidenten wurden auch R. Różycki, W. Orski und S. Łaszewski ernannt, wobei nur Letzterer - als Spezialist für das preußische Recht - nicht zuvor am Wiener VwGH tätig

\footnotetext{
${ }^{12}$ Auf Antrag des Ministerratspräsidiums berief das Staatsoberhaupt am 29. 8. 1922 J. Sawicki in die Funktion als Präsident des Obersten Verwaltungsgerichtshofes. In der Antragsbegründung hieß es: „Das solide juristische Wissen und seine außerordentlichen Fähigkeiten, die er in sämtlichen ihm übertragenen Aufgaben bewies, sowie seine langjährige Erfahrung und die Arbeit als Richter, Senatspräsident und Präsident am Wiener Verwaltungsgerichtshof qualifizieren ihn besonders für die Position des Präsidenten am Obersten Verwaltungsgerichtshof"; AAN, Prezydium Rady Ministrów, Bd. 19, Mikrofilm Nr. 20063, 577.
} 
gewesen war. Von den 22 Richtern der ersten Vollversammlung stammte über die Hälfte aus dem zuvor habsburgischen Teil Polens. Jan Sawicki blieb bis zu seiner Pensionierung aus politischen Gründen am 7. Februar 1928 Präsident [Pierwszy prezes], worauf R. Różycki diese Pflichten für zwei Jahre interimistisch übernahm und 1930 schließlich auch zum Präsidenten ernannt wurde. Er behielt diesen Posten bis zu seiner Pensionierung 1932. Seit November 1926 war er überdies Präsident des Kompetenzgerichtshofes.

Nach R. Różycki wurde J.K. Piętak neuer Gerichtshofpräsident. Er erfreute sich bei der Regierung großen Vertrauens und bereitete u.a. die Vorlage für ein neues OVwGH-Gesetz vor, das bei der Reform des Gerichtshofes in eine ganz andere Richtung abzielte, als von R. Różycki geplant. Dieses neue Gesetz von 1932 regelte System und Aufgaben des Gerichtshofes kaum anders, allerdings wurden der Regierungseinfluss bei der Postenbesetzung erheblich und auch die Kompetenzen des Präsidiums erweitert. Różycki dagegen plante eine grundlegende Gesetzesänderung durch die Einrichtung untergeordneter Verwaltungsgerichte. ${ }^{13}$ Die Regierung bereitete, trotz ursprünglicher Akzeptanz für eine Vorbereitung der Reform durch R. Różycki, heimlich eine eigene Vorlage für ein neues OVwGH-Gesetz vor, das im Grunde den Rechtsstatus eines Gerichtshofes beibehielt. Die Vermutung vorrangig politischer Gründe hierfür liegt nahe. Nach dem Maiputsch von 1926 entschloss sich die Regierung (Sanacja) ab 1930 zum offenen Kampf gegen die Opposition, einschließlich der Verhaftung und Inhaftierung

\footnotetext{
${ }^{13}$ Er offenbarte dies in einem Gespräch mit Premier Kazimierz Świtalski im Mai 1929: „Różycki zeigt sich von den vermehrten Eingängen beim Verwaltungsgerichtshof beunruhigt und plant daher die Einrichtung von zweitinstanzlichen Verwaltungsgerichten. [...] Er möchte, dass vier Gerichte auf Wojwodschaftsebene entstehen. Ich sagte ihm, dass ich in der Sache theoretisch vorfühlen könne"; siehe: ŚWITALSKI, Diariusz 427.
}

politischer Gegner. Sie war also in keiner Weise an einer verstärkten Kontrolle des Staatsapparates durch die Verwaltungsgerichte interessiert.

Diese Umstände dürften Różyckis Entschluss zum Pensionsantritt beschleunigt haben; an sich hätte seine Amtszeit erst 1936 geendet. Vielleicht wurde er hinter den Kulissen auch wegen seines fortgeschrittenen Alters, 1932 war er bereits 75, dazu gezwungen, nachdem seine Pensionierung zeitgleich mit dem Inkrafttreten des neuen OVwGH-Gesetzes und der Ernennung des neuen Präsidenten Jan K. Piętak verlief. Dieser wechselte aus der Kanzlei des Premiers in diese Funktion und hatte nicht ganz ein Jahr die Leitung inne. Sein plötzlicher Tod zwang die Regierung, sich nach einer anderen vertrauenswürdigen Persönlichkeit umzusehen. Bevor diese gefunden werden konnte, war Włodzimierz Orski (1933-1934) fast ein Jahr Präsident des Obersten Verwaltungsgerichtshofes. Der letzte Vertreter der ehemaligen Richter des vormals österreichischen VwGH war demnach auch ein Übergangspräsident.

\section{Eingeschränkte Unabhängigkeit des Obersten Verwaltungsgerichtshofes} Die Absetzung von Jan Sawicki nach dem
Maiputsch von Marschall Józef Piłsudski 1926

Der Oberste Verwaltungsgerichtshof setzte sich aus Richtern mit höchstem Fachwissen sowie Erfahrung in der Rechtsprechung zusammen und erwarb sich in Kürze den Ruf des wichtigsten Wächters der Rechtsstaatlichkeit. Besonders seine Unabhängigkeit wurde geschätzt, die sich u.a. darin ausdrückte, dass fiskalische Interessen nicht über den Bürgerrechten standen. Der Gerichtshof galt also als taub für Einflüsterungen von Regierungsseite. Davon konnten sich die Regierungen des Sanacja-Systems, die nach dem Militärputsch von 1926 an der Macht waren, überzeugen. Illegale Praktiken der neuen 
Machthaber wurden seitens der Höchstrichter abgestraft. Ein spektakuläres Beispiel für das gesetzlose Vorgehen der neuen autoritären Machthaber war die illegale Berufung des Generalwahlkommissärs vor den Wahlen 1928 durch das Staatsoberhaupt: Nach dem Gesetz hätte der Präsident einen von drei Kandidaten, die vom Präsidialkollegium der Höchstgerichte vorgeschlagen wurden, auszuwählen gehabt. Marschall Piłsudski beschloss dagegen, dass sein Vertrauensmann Stanisław Car, der damalige Vizejustizminister, Kommissär werden sollte. ${ }^{14}$

Die Regierung hatte daher diverse Bemühungen unternommen, damit dieser in den Dreiervorschlag aufgenommen würde. Für die Machtelite nach 1926 war der Wunsch des Marschalls nämlich oberstes Gesetz. Diese Auffassung teilten die Präsidenten der Höchstgerichte jedoch nicht. Sie erkannten, in Verteidigung des Rechtsstaates, dass die Berufung eines Regierungsmitgliedes als Wahlkommissär dem Geist des Gesetzes widerspräche und beugten sich dem Druck nicht, weshalb sich Car nicht im Dreiervorschlag

${ }^{14} \mathrm{~S}$. Car hatte insofern Vorbehalte gegenüber Piłsudskis Entscheidung, da er die begonnen Arbeit im Justizministerium liegen lassen musste, sah aber naturgemäß kein Hindernis in der Übernahme der neuen Funktion und glaubte auch nicht, dass es diesbezüglich Schwierigkeiten geben würde. K. Świtalski war zu der Zeit von Regierungsseite verantwortlich für die Wahl und stellte ein Gespräch mit S. Car dar: „Er sagte mir, dass, wenn der Kommandant (Pilsudski, Anm. des Autors) es wünsche, er diese Funktion übernehmen würde und sich das in formaler Hinsicht machen ließe, da unter den vier Kandidaten für das Präsidium er, Car, mit Mogilnicki und Pohorecki reden könnte und Meysztowicz mit Seyda. Bliebe nur noch Dworski, bei dem Einflussnahme schwierig sei." Dieses Gespräch illustriert anschaulich die Mentalität der neuen Machtelite, die Menschen und das Recht unter instrumentellen Gesichtspunkt betrachten. Das Ziel der Regierung war eindeutig: Einfluss auf den Verlauf der freien Wahlen gewinnen und den Einfluss der Opposition zurückdrängen. Darüber waren sich auch die Höchstrichter im Klaren und daher rührte ihr entschlossener Widerstand; siehe: ŚWITALSKI, Diariusz 269. wiederfand. Trotzdem betraute ihn der Staatspräsident auf Antrag der Regierung mit der Funktion des Generalwahlkommissärs, und eine Reaktion ließ nicht lange auf sich warten: Am 6. Februar 1928 erließ der Staatspräsident im Wege einer Rechtsverfügung mit Gesetzeskraft ein neues Gesetz zur allgemeinen Gerichtsverfassung, kraft dessen, innerhalb von drei Monaten nach Inkrafttreten, Richter an Höchstgerichten ohne ihre Zustimmung in den Ruhestand versetzt werden konnten. Anfang 1929 wurden so acht Höchstrichter entfernt, darunter zwei Präsidenten, die im Jahr zuvor gegen die Regierung aufgetreten waren. ${ }^{15}$

Das erste Opfer dieser Säuberungen in der Gerichtsbarkeit wurde jedoch Jan Sawicki. Am 7. Februar 1928 ergänzte der Präsident das Gesetz zur allgemeinen Gerichtsverfassung um eine Verfügung über eine Abänderung der Bestimmungen über den Obersten Verwaltungsgerichtshof, die am selben Tag in Kraft trat. Sie ermöglichte, auch Richter dieses Gerichtshofes in den Ruhestand zu versetzen. Noch am gleichen Tag wurde auf persönlichen Antrag von Józef Piłsudski, der zu dieser Zeit Premier war, Jan Sawicki vom Staatspräsidenten im Eilverfahren vom Vorsitz am OVwGH enthoben. Die Verfügung trat mit Verlautbarungsdatum in Kraft; offensichtlich nur, um sich des Gerichtshofpräsidenten möglichst rasch $\mathrm{zu}$ entledigen. Noch am gleichen Tag wurde im Ministerrat die Zustimmung eingeholt, dass der Premier vor dem Staatspräsidenten wegen der Absetzung von J. Sawicki vorstellig werden dürfe, obwohl für diesen Tag gar keine Ministerratssitzung formell anberaumt gewesen war. Ebenfalls am

\footnotetext{
${ }^{15}$ Władysław Seyda, der Präsident des Obersten Gerichtes, wurde ebenfalls in den Ruhestand versetzt. Als ehemaliger Funktionär der Nationaldemokraten war er politisch nicht genehm. Ebenso erging es dem Senatspräsidenten der Strafkammer Aleksander Mogilnicki; siehe: WITKOWSKI, NAWORSKI, Kompetencje głowy państwa $22 f$.
} 
gleichen Tag wurde Sawicki vom Staatspräsidenten offiziell in den Ruhestand versetzt. Sämtliche rechtlich und formal notwendigen Schritte zur Absetzung von Sawicki wurden innerhalb eines Tages erledigt!

Diese Eile, mit der Sawicki seiner Stellung verlustig ging, mag verwundern. ${ }^{16}$ Leider lässt das Quellenstudium die Beantwortung nach dem direkten Anlass, der Piłsudski unter Ausnutzung des neuen Gesetzes zur allgemeinen Gerichtsverfassung zu einer sofortigen Abberufung Sawickis veranlasste, nicht zu. Auch die SejmDebatte im Anschluss (März 1928) bringt hierüber keinen Aufschluss. Ein Vertreter des nationalen Lagers (Nationaldemokraten - Narodowa Demokracja) beantragte, jenen Artikel, der ermöglichte, Richter in den Ruhestand zu versetzen, aufzuheben. Dabei berief er sich auf die

${ }^{16}$ AAN, Prezydium Rady Ministrów, Bd. 41, Mikrofilm Nr. 20081, 630-631: „Warschau, den 7. Februar 1928. Folgende Minister äußerten mir gegenüber am heutigen Tag Zustimmung für den Antrag des Vorsitzenden des Ministerrates und Militär[!]ministers bezüglich der Versetzung in den Ruhestand von Jan Sawicki, dem Präsidenten des Obersten Verwaltungsgerichtshofes: der Innenminister, der Finanzminister, der Justizminister, der Kultus- und Bildungsminister, der Industrie- und Handelsminister, der Kommunikationsminister, der Minister für öffentliche Arbeiten, der Minister für Arbeit und Wohlfahrt, der Minister für die Landwirtschaftsreform. Unterschrift - Sekretär des Ministerrates. Ich bestätige, wie aus dem Obengenannten hervorgeht, dass der Ministerrat mit heutigem Tage dem obigen Antrag stattgibt. Unterschrift - Der Vorsitzende des Ministerrates 7. Februar 1928. Der Antrag des Vorsitzenden des Ministerrates in Sachen Versetzung in den Ruhestand von Jan Sawicki, Präsident am Obersten Verwaltungsgerichtshof. Nach Art. $284 \S 1$ Verfügung des Präsidenten der Republik Polen vom 6.2.1928, dem Allgemeinen Gerichtsverfassungsgesetz und Art. 1, 11, 12 und 13 der Verfügung des Präsidenten der Republik Polen vom 7. Februar 1928 über die Änderung und Ergänzung der Vorschriften betreffs der Verwaltungsgerichtshofgesetzes sowie der Dienstverhältnisse der Richter am OVwGH beantrage ich, dass der Präsident der Republik Polen den Präsidenten des OVwGH, Jan Sawicki, in den Ruhestand versetzten möge". desaströse öffentliche Meinung nach der Absetzung des Gerichtshofspräsidenten, führte allerdings auch keine konkreten Gründe für diese Absetzung an und deutete an, dass es sich um Vergeltung für die Verteidigung der richterlichen Unabhängigkeit gehandelt habe. ${ }^{17}$ Vielleicht war der Meinungsunterschied bei der Berufung des Wahlkommissärs ursächlich, in der sich der Gerichtshofpräsident regierungskritisch geäußert haben könnte.

Zwischen Gerichtshof und Regierung kam es auch hinsichtlich der Überprüfung der Rechtmäßigkeit von Verfügungen mit Gesetzeskraft, die vom Staatspräsidenten erlassen worden waren, zu Kontroversen. Trotz gegenteiliger Regierungsmeinung erkannte der Gerichtshof letztlich auf Zuständigkeit bei der Überprüfung. Jedenfalls trat J. Sawicki offen gegen versuchte Einflussnahmen der Regierung auf die Judikatur des Gerichtshofes auf. In dieser Frage war die

${ }^{17}$ Der Abgeordnete Jan Pieracki vom Sejm-Klub der Volksnationalen Vereinigung [Zwiazek LudowoNarodowy] brachte einen Antrag auf Aufhebung von Art. $284 \S 1$, auf dessen Grundlage J. Sawicki entfernt wurde, ein. Vgl. das Stenogramm der 6. Sejm-Sitzung vom 31. 3. 1928. Dieser Standpunkt des Klubs wurde von der Linken unterstützt, da sich die gesamte Opposition einig war im Widerstand gegen die Beschränkung der richterlichen Unabhängigkeit. Vgl. das Stenogramm der 36. Sejm-Sitzung vom 14. 10. 1928. Schließlich gelang es mittels Gesetzesänderung, die Einflussmöglichkeiten auf die Versetzung in den Ruhestand ohne Zustimmung zu beschränken. Dass die Rechte im Sejm nach der Absetzung von Sawicki auf den Plan gerufen wurde, mag der Vermutung geschuldet sein, dass Sawicki als Katholik mit der Nationaldemokraten sympathisiert haben dürfte, und darin der Grund für seine Pensionierung zu suchen sei; ähnlich wie im Fall des Präsidenten des Obersten Gerichtes W. Seyda. Auf diesen wurde nach 1926 Druck ausgeübt, er möge auf seine Funktion verzichten. Die rechtsgerichtete "Głos Narodu" [Stimme der Nation] kommentierte die Absetzung mit einer kurzen, jedoch aussagekräftigen Notiz: „Hr. Sawicki aus dem Weg geräumt", als hätte man dies im nationalen Lager so erwartet; siehe: Głos Narodu, Nr. 42 vom 11. 2. 1928. 
Haltung seines Nachfolgers R. Różycki elastischer, der in Sachen von gesamtgesellschaftlichen Auswirkungen die Regierungserwartungen sondierte. ${ }^{18}$

Natürlich ging es den politischen Funktionären aus dem Piłsudski-Lager hauptsächlich darum, jene Personen aus Führungspositionen im Gerichtswesen $\mathrm{zu}$ entfernen, die aufgrund ihrer Unabhängigkeit die Realisierung politischer Ziele der Sanacja-Formation nicht gewährleisteten, und um die Besetzung dieser Posten mit regierungsfreundlichen Ersatzleuten. Diesbezüglich hatte der Justizminister keine Bedenken: Er enthob zwar J. Sawicki seiner Stellung, obwohl er selbst nicht dem politischen Umfeld von Piłsudski angehörte. Nach seinem Rücktritt nach zweijähriger Amtszeit erklärte er jedoch: „Ich hatte auch keine abschreckenden Exempel in der Verwaltung und umso weniger im Gerichtswesen im Sinn. Ich sehe das so, dass sich die Regierung auf treu ergebene Beamte stützen können muss; Obwohl die Regierung des Marschalls das Gros der Nation vereinigen konnte und nicht bloß auf die 1. Brigade angewiesen war, gab es für die Übernahme sämtlicher Verwaltungspositionen, ganz zu schweigen von den Richterposten, zu wenige und außerdem vollkommen unvorbereitete Personen. Wenn ich gewusst hätte, dass es zu Gesetzesbruch und Einflussnahme kommen würde, hätte ich das Portefeuille nicht angenommen". ${ }^{19}$

\footnotetext{
${ }^{18}$ R. Różycki versuchte 1929 in einem Gespräch mit dem damaligen Premier K. Świtalski noch, die Zustimmung der Regierung für die eingeschlagene Rechtsprechung zu erlangen. Świtalski notierte: „Er fragte, ob die Auflösung der deutschen Vermögen rigoros durchzuführen sei. Ich stimmte ihm $\mathrm{zu}^{\prime \prime}$, in: ŚWITALSKI, Diariusz 427.

${ }^{19}$ GARLICKI, Sądne dni. Diese Aussage von Aleksander Meysztowicz (Justizminister 1926-1928), einem Vertreter der konservativen Seite, sollte ein Gegengewicht zu den Nationaldemokraten sein, die sich nach 1926 in Opposition zur Sanacja-Regierung befanden. Er war also einerseits für die Machthaber weltanschaulich zweifelhaft, andererseits allerdings
}

\section{Der Bedeutungsverlust der Richter österreichisch juristischer Provenienz im Obersten Verwaltungsgerichtshof Die Gerichtshofleitung in Händen der Verwaltungs- und Regierungskreise}

Mit der Verfügung des Staatspräsidenten vom 27. Oktober 1932 über die neuen Regelungen zur Richternominierung beginnt ein neuer Abschnitt für den OVwGH. Zweck dieser Verfügung war, dem Vorsitzenden im Ministerrat das ausschließliche Vorschlagsrecht für Kandidaten in Führungspositionen am Gerichtshof vorzubehalten. Ein Kandidat für die Präsidentschaft musste nicht einmal mehr Richter am Gerichtshof sein! Diese Übergangsbestimmungen ermöglichten der Regierung, unliebsame Personen durch Sanacja-genehme zu ersetzen. Einschränkend muss allerdings hinzugefügt werden, dass in der Praxis sehr beschränkt davon Gebrauch gemacht wurde.

Ein Beispiel hierfür war etwa die Ernennung von Jan L. Piętak zum Präsidenten des Obersten Verwaltungsgerichtshofes auf Basis dieser neuen Vorschriften. Er war zuvor der Leiter des Rechtsbüros für den Vorsitzenden des Ministerrates und wurde u.a. für sein Talent geschätzt, „Rechtsvorschriften an die Lebensbedürfnisse anzupassen". ${ }^{20}$ Seines Amtes konnte er sich je-

politisch nützlich. Daher wurde auch der PilsudskiVertraute S. Car Vizeminister, um gleichermaßen Meysztowicz ",auf die Finger zu schauen“. Nach der Wahl löste er dann auch Meysztowicz als Justizminister ab.

${ }^{20}$ Eine treffende Beschreibung von J.K. Piętak stammt vom Vizeminister für Kultus und Bildung, dem Geistlichen Prof. Bronisław Żongołłowicz: „Aufgeweckt und klug. Er war fexibel in der Anpassung der Rechtsnormen an die Alltagsbedürfnisse, bzw. beim Aufpropfen selbiger auf die Rechtsnormen mittels Interpretation. Kompromissbereit, nachgiebig, gutherzig und gutmütig. Ein Vertreter des guten alten galizischen Beamtentyps"; siehe: ŻonGOŁŁOWICZ, Dzienniki 481. 
doch nicht lange erfreuen; wie erwähnt verstarb er kurz nach der Ernennung. 1934 übernahm der ebenfalls regierungsnahe Bronisław Hełczyński das Präsidentenamt am Gerichtshof. Er war seit 1928 Leiter der sogenannten Zivilkanzlei des Staatspräsidenten und seine steile Karriere war bezeichnend für loyale Beamte aus dem Umfeld der Sanacja.21 Hełczyński blieb bis zum Ausbruch des Zweiten Weltkriegs Präsident des Obersten Verwaltungsgerichtshofes, womit sich nicht nur durch Erreichung des Pensionsalters der Einfluss der ehemaligen Richter am Wiener Verfassungsgerichtshof verringerte. Ausschlaggebend hierfür waren vor allem die Folgen der politischen Veränderungen nach 1926.

${ }^{21}$ Ganz anders als J.S. Piętak wird B. Hełczyński vom oben erwähnten B. Żongołlowicz umrissen: „Dieser Präsident des Obersten Verwaltungsgerichtshofes ist von unglaublicher Dreistigkeit. Für seine Habilitation wurde er nicht nur Dozent, sondern gleich o.Prof. an der Jagiellonen-Universität; für ein paar Jähren Repräsentationsaufwand im Schloss vom Chef der Zivilkanzlei wurde er Präsident des Obersten Verwaltungsgerichtshofes und weil er nie in einer polnischen Auslandsschule war, weiß er natürlich über unsere Auslandsschulen Bescheid. Was ihn zur Professur und Präsidentenfunktion qualifiziert? Frechheit und Protektion. In der Sprache der Sanacja-Vasallen heißt das dann „Mensch des Regimes“. Der klassische Büttel jedweden „Regimes”; siehe: ŻongOŁŁOWICZ, Dzienniki 613.

\section{Korrespondenz:}

Dr. hab. Andrzej Dziadzio

Uniwersytet Jagiellonski

Katedra Powszechnej Historii Państwa i Prawa UJ

ul. Gołębia 9

31-007 Kraków

andrzej.dziadzio@uj.edu.pl

\section{Abkürzungen:}

$\begin{array}{ll}\text { AAN } & \text { Archiwum Akt Nowych } \\ \text { Dz.U. } & \begin{array}{l}\text { Dziennik Ustaw } \\ \text { (polnisches Gesetzblatt) }\end{array} \\ \text { OVwGH } & \text { Oberster Verwaltungsgerichtshof }\end{array}$

\section{Literatur:}

Andrzej DzIADZIO, Die Angelegenheiten aus Galizien in der Rechtsprechung der österreichischen Verwaltungsgerichtsbarkeit im 19. und 20. Jahrhundert, in: Zoran POKROVAC (Hg.), Rechtsprechung in Osteuropa. Studien zum 19. und frühen 20. Jahrhundert. Rechtskulturen des modernen Osteuropa. Traditionen und Transfers (=Veröffentlichungen des Max-Planck-Instituts für Rechtsgeschichte 6.2, Frankfurt am Main 2012) 475-540.

Andrzej GARLICKI, Sądne dni, Polityka vom 28. 05. 2007.

Stanisław GoŁĄB, in: Czasopismo Prawnicze i Ekonomiczne 25 (1929) 1-12.

Andrzej GulcZYŃSKI, Die für die Anwendung des deutschen Privatrechts zuständigen Mitglieder des polnischen Obersten Gerichtshofes zwischen 1920 und 1939, in: Woiciech DAJCZAK, Hans-Georg KnOTHE (Hgg.), Deutsches Sachenrecht in polnischer Gerichtspraxis (= Schriften zur Europäischen Rechts- und Verfassungsgeschichte 49, Berlin 2005) 77-134.

Josef KASERER, Das Gesetz vom 22. October betreffend die Errichtung eines Verwaltungsgerichtshofes [...] mit Materialien (Wien 1876).

Dorota MALEC, Najwyższy Trybunał Administracyjny 1922-1939 w świetle własnego orzecznictwa (Kraków 1999).

Dorota MALEC, Der Oberste Verwaltungsgerichtshof und seine Rechtsprechung in den Jahren 1922 1939, in: Zoran POKROVAC (Hg.), Rechtsprechung in Osteuropa. Studien zum 19. und frühen 
20. Jahrhundert. Rechtskulturen des modernen Osteuropa. Traditionen und Transfers (=Veröffentlichungen des Max-Planck-Instituts für Rechtsgeschichte 6.2, Frankfurt am Main 2012) 541-596.

Thomas OlechOWSKI, Europäische Modelle der Verwaltungsgerichtsbarkeit im 19. Jahrhundert, in: Martin POLASCHEK, Anita Ziegerhofer (Hgg.), Recht ohne Grenzen. Grenzen des Rechts (Wien 1998) 137-149.

Jan SAWICKI, Sądy administracyjne w Polsce, in: Dziennik Urzędowy Ministerstwa Sprawiedliwości (dział nieurzędowy) (1920) 178-188, 234-240.

Kazimierz ŚWITALSKI, Diariusz 1919-1935 (Warszawa 1992).

Anna TARNOWSKA, Sądownictwo administracyjne II RP a pruski model sądownictwa administracyjnego. in: Studia z Dziejów Państwa i Prawa Polskiego 9/2 (2006) 414-444.
Anna TARNOWSKA, Die niedere Verwaltungsgerichtsbarkeit im Polen der Zwischenkriegszeit. Die Rechtsprechung der Woiwodschaftsverwaltungsgerichte im ehemals preußischen Landesteil, in: Zoran POKROVAC (Hg.), Rechtsprechung in Osteuropa. Studien zum 19. und frühen 20. Jahrhundert. Rechtskulturen des modernen Osteuropa. Traditionen und Transfers (=Veröffentlichungen des Max-Planck-Instituts für Rechtsgeschichte 6.2, Frankfurt am Main 2012) 619-674.

Zbigniew WITKOWSKI, Zbigniew NAWORSKI, Kompetencje głowy państwa $\mathrm{w}$ zakresie mianowania na stanowiska w II Rzeczypospolitej (ze szczególnym uwzględnieniem obsady stanowisk sędziowskich), in: Przegląd Sejmowy XVI, 4(87) (2008) 9-30.

Bronislaw ŻongOŁŁOWICZ, Dzienniki 1930-1936 (Warszawa 2004) 481. 